Itinéraires Itinéraires

Littérature, textes, cultures

\title{
Mythification historique et prolifération identitaire dans le biopic Evita
}

The Mythologizing of History and the Proliferation of Identities in the Biopic Evita

\section{Gabriel Laverdière}

\section{OpenEdition}

\section{Journals}

Édition électronique

URL : http://journals.openedition.org/itineraires/4070

DOI : 10.4000/itineraires.4070

ISSN : 2427-920X

\section{Éditeur}

Pléiade

\section{Référence électronique}

Gabriel Laverdière, "Mythification historique et prolifération identitaire dans le biopic Evita », Itinéraires [En ligne], 2017-1 | 2018, mis en ligne le 25 juin 2018, consulté le 19 avril 2019. URL : http:// journals.openedition.org/itineraires/4070 ; DOI : 10.4000/itineraires.4070

Ce document a été généré automatiquement le 19 avril 2019

\section{$\circledast \oplus \Theta \Theta$}

Itinéraires est mis à disposition selon les termes de la licence Creative Commons Attribution - Pas d'Utilisation Commerciale - Pas de Modification 4.0 International. 


\title{
Mythification historique et prolifération identitaire dans le biopic Evita
}

The Mythologizing of History and the Proliferation of Identities in the Biopic Evita

\author{
Gabriel Laverdière
}

Tout récit est, par définition, infidèle. Martínez ([1995] 1997 : 82)

1 Le cinéma compte plusieurs récits prétendument biographiques ou imaginés d'après la vie de personnalités connues. Puisque la durée d'un film est généralement restreinte et qu'il procède à un découpage spatio-temporel, puis à un assemblage, il ne peut offrir qu'une version réduite de l'histoire d'une vie, et la fictionnalisation à laquelle cette dernière est soumise contribue à la création d'une autre histoire, narrative et imaginaire, que le spectateur prolonge (ou synthétise) en pensée. Inversement, voire corrélativement, comme le suggère Lucie Roy (1999: 22-23), le cinéma a tendance à augmenter la réalité : par l'acte de la représentation, «le "réel" est [d'abord] diminué, coupé de son cours temporel et de son continuum spatial [mais] il peut être [...] agrandi par des mouvements de caméra, des gros plans, etc. [...] tiré de l'anonymat [...] de la quotidienneté, le "réel" se trouve [...] agrandi [...]». À cette activité, qui est la poétique du film, s'ajoute la fictionnalisation de l'histoire, dont Paul Ricœur a profondément discuté. Qu'il soit documenté (un film à caractère historique ou dont la valeur semble être la reproduction d'une période, d'un événement) ou biographique (le biopic), un film adopte le plus souvent les modalités esthétiques de la fiction. En vertu de cet « ouvragement » du film (Roy 2015 : 222), on peut concevoir que le cinéma concoure à une forme de mythologisation de l'histoire.

2 Si cela paraît relever de l'évidence, la correspondance entre réalité biographique (monde de référence) et représentation filmique (réalité esthétique) ne cesse de préoccuper, ou de fasciner, les spectateurs. À cet égard, le film Evita (Alan Parker, 1996) offre l'occasion 
d'examiner ce qu'on estime ordinairement être le problème du biopic : sa «fidélité ». Il annonce le biopic "postmoderne", pour lequel les stratégies classiques du genre sont écartées ou reportées. Il montre que cette correspondance entre réalité et représentation n'est qu'approximative, qu'elle dépend de conventions, et que, donc, la vérité du biopic se trouve autre part.

Le film de Parker relâche les rênes de la biographie habituelle en épousant l'esthétique frontalement spectaculaire de la comédie musicale. Et il intègre à la représentation une figure qui est aussi spectaculaire, la star Madonna, qui a multiplié, dans sa carrière de chanteuse, les identités de toutes sortes. Le film prend pour prétexte narratif la vie d'Eva Perón, mais il participe aussi, en sous-main, de l'écriture de l'autre récit, de l'autre identité narrative, celle de son interprète. La perception qu'aura le spectateur de cette double écriture ne saurait lui permettre de retenir la vérité historique en un sens absolu. D’ailleurs, la vie de Perón compte elle-même de nombreuses approximations. La vraie Eva Perón a bien existé, mais on peut difficilement séparer statut historique et statut mythique, comme l'attestent des versions alternatives de sa biographie; la vraie Madonna existe, mais son statut est d'emblée mythifié par l'épaisseur culturelle de son œuvre médiatique (images, chansons, films, etc.). En ce qui concerne ces deux femmes, le film invite à concevoir la " mitoyenneté » de leur identité entre personne et personnage (Roy 2015 : 150) ; il s'avère alors un de ces « cas paradoxaux » qu'évoquait Ricœur ([1990] 1996 : 160). Le travail du film, sur le mode de la fiction spectacularisante, encourage la mythification des figures qu'il met en intrigue. Il éloigne la vérité historique absolue en nous rappelant que celle-ci n'est pas pleinement libre d'une interprétation et d'une compréhension des faits, d'une signifiance. Le film incite à penser que le biopic risque d'informer sur l'imaginaire de sociétés, ou encore sur les apories de l'identité et sur la sorte de résolution que permet le récit, comme le proposait Ricœur, davantage qu'il ne risque d'informer sur la réalité historico-biographique, à laquelle, néanmoins, il s'alimente.

4 Cela dit, la fictionnalisation ne soustrait pas les films à une interrogation historique. Il faut, pour le concevoir, rebrousser le chemin de la science historique et retrouver la valeur du récit mythique, que Mircea Eliade $(1969: 9,15)$ définit de la sorte :

Au lieu de traiter [...] le mythe dans l'acception usuelle [...] en tant que [...] «invention», «fiction», [certains] l'ont accepté tel qu'il était compris dans les sociétés archaïques, où le mythe désigne [...] une " histoire vraie » [...], exemplaire et significative. [... Il] relate un événement qui a eu lieu dans le temps [...] des " commencements » [...]. C'est [...] le récit d'une « création » [...].

Il importe de considérer les arts « de l'imaginaire » comme les révélateurs des sociétés et des mentalités, d'autant plus que les pratiques culturelles se sont industrialisées, qu'elles se sont démocratisées (ceci grâce à cela), et que les écritures de l'image font partie des formes privilégiées de représentation. Les images contiennent souvent des récits. Ces récits, la plupart du temps fictionnels au cinéma, s'apparenteraient à des formes mythiques modernes.

6 Au mythe correspondent ainsi deux significations en apparence concurrentes, mais qui s'appuient en fait l'une sur l'autre: le mythe révélateur et fondateur d'états de monde, puis le mythe comme récit fallacieux. Le mythe dit que la réalité doit faire l'objet d'une saisie, par l'assignation d'un sens, et qu'elle ne se donne pas à travers de simples représentants. Le cas Evita le démontre, à commencer par la biographie de Perón ellemême, dont l'ambiguïté a été cultivée de longue main. 


\title{
Le mythe Perón
}

\author{
Peu à peu, Evita se transforma en une légende [...]. \\ Elle cessa d'être ce qu'elle avait dit et fait pour \\ devenir les propos et actes qu'on lui attribuait. \\ Martínez (1997 : 16)
}

7 La vie d'Eva Duarte de Perón (1919-1952), seconde femme de Juan Perón (en 1945), demeure un mystère quant à des faits qu'il s'agirait d'établir. S'il se conçoit qu'un puissant leader invite à des lectures biographiques diverses, le cas d'Evita est réputé complexe. On lui reconnaît des réalisations: elle pave la voie pour les femmes en politique en occupant une fonction d'État, en les mobilisant par le Parti péroniste féminin (Partido Peronista Femenino) et en appuyant leur droit à voter aux élections. On lui reproche son apport aux pratiques oppressives du gouvernement (répressions violentes et systémiques). Souhaitant relever les faits concernant sa vie, ses opposants comme ses partisans admettent ce qui suit. Elle est née d'une liaison extraconjugale dans un village très pauvre. Enfant illégitime (comme le veut l'expression consacrée) et victime de préjugés sociaux, elle partit à 15 ans pour Buenos Aires et devint une actrice connue. Elle rencontra Juan Perón en 1944, apprit la politique à l'usage et gagna la faveur populaire tout en s'attirant les foudres des classes moyennes ou supérieures et des antipéronistes. Elle mourut d'un cancer au faîte de sa gloire à 33 ans, en 1952.

De ce récit biographique, le sens du personnage est exclu. Divers lecteurs en ont offert des variations, lui attribuant des intentions, des raisonnements, produisant ainsi du sens à partir de faits (avérés, allégués, litigieux ou controuvés), à commencer par sa première biographe notable, Mary Main. Dès 1952, la biographe publie The Woman with the Whip, dont le titre figure la thèse de l'auteure ${ }^{1}$ et que d'aucuns considèrent comme " un recueil des plus virulents potins antipéronistes ${ }^{2} »$ (Fraser et Navarro 1996 : 199). Main y dénonce le régime de Perón, décrit comme une dictature. Signalant la collaboration d'Evita, Main ([1952] 1980 : 66-71, 215-221; Flores $1953: 63-69,207-213)$ dépeint son ascension menée par une ambition opportuniste. Sans argent, talent, instruction ni contacts, Eva se serait stratégiquement élevée par la manipulation d'autrui. Main attribue cette attitude à la situation des femmes de l'époque: "Que pour réussir Eva utilisât ses amis comme échelons et sa féminité comme une arme, voilà qui semble naturel étant donné son milieu familial et les mœurs de son pays. La femme argentine avait été exploitée autant que le péon [...]» (Flores 1953: 32-33; Main [1952] 1980: 36). Bien qu'elle situe Evita relativement aux défis imputables à sa classe sociale et à son sexe, Main ([1952] 1980 : 98 ; Flores 1953 : 95) ne manque pas pour autant de décrire ses méthodes comme brutales et sa fondation comme une entreprise fantasque de mésusage des fonds publics; la fascination qu'elle inspirait et la propagande qui la célébrait masquaient ses agissements répréhensibles. Issue du peuple, elle incarnait le rêve inouï de prospérité que partageaient les descamisados (sans chemises de la classe ouvrière), qu'elle courtisait infatigablement. Notons que, si des remarques sur le mode de vie argentin de l'époque authentifient ses propos, Main cite rarement ses sources et emploie, à titre de référence, des locutions vagues comme "on raconte que ", ce qui incite à douter de certaines affirmations (Main [1952] 1980 : 77, 231, 236 ; Flores 1953 : 75, 221, 224).

Deux identités : Santa Evita, désintéressée et déterminée à améliorer le sort de la classe ouvrière, ou la "Femme au fouet", une démagogue fasciste qui s'est enrichie au 
détriment des pauvres. Dans la même figure historique clivée, chaque identité est la doublure de l'autre. Cette coexistence paradoxale rend compte de la relativité de la vérité historico-biographique et de ce que des sociétés s'approprient les personnalités publiques. Les récits associés à ces personnes devenues personnages peuvent être investis d'une propriété mythique, et même, dans le cas d'Evita, d'une aura mystique, qui d'ailleurs fut ouvertement promue. On a si souvent eu recours à son nom, à sa légende, son histoire fut si souvent réinventée qu'ont émergé de multiples Evita. Dans son étude du film, Savigliano (2002 : 357) remarque que les Argentins ont perpétué la diversification des histoires et mythes associés à Evita. Le film de Parker s'inscrit ainsi dans une tradition de réécritures : sa vie a inspiré des films, romans, spectacles, biographies, qui alignent faits et fabrications, de sorte que la véritable Evita demeure un mystère. Preuve de la mythification dont elle a été l'objet, son cadavre a servi, de façon abjecte, à des fins politiques $^{3}$ : Evita était moins humaine qu'un symbole, réinvesti suivant les besoins des uns et des autres. Elle est devenue une figure mythique, surtout pour les péronistes, un Être suprême. Ce statut repose, certes, sur la progression fulgurante d'Evita et sur ses actions qui ont transformé la société. Cela dit, tous les aspects de sa vie ont été augmentés d'une puissance affective populaire, entretenue par l'imaginaire collectif et par des pratiques promotionnelles ou représentationnelles (des effigies).

10 Selon Eliade (1963: 15), "le mythe raconte comment, grâce aux exploits des Êtres Surnaturels, une réalité est venue à l'existence [...]». L'Argentine de la fin des années 1940 s'est construite entre autres par le concours de la femme politique, mais on ne saurait dissocier sous tous les rapports ces faits des récits qu'ils ont inspirés. Qu'Evita soit adorée ou détestée, il parait impossible de raconter sa vie sans que ses propriétés mythiques ne soient reconnues. On tient ces propriétés pour réelles, car elles ont contribué à l'interprétation des faits, au développement national, à la conceptualisation de l'histoire du pays, dans la mesure où Evita a valu pour symbole aux yeux de plusieurs : "[...] chacun déployait dans ce corps [embaumé et exposé] les recoins de sa propre mémoire ", écrit Martínez (1997 : 16). Le film de Parker puise à ce fonds mythographique et y retourne ses propres refigurations spectacularisées. D’après Savigliano (2002: 357), l'effet du film durera et la réécriture qu'il propose affectera "la représentation et l'interprétation de l'histoire argentine ».

\section{Le spectacle de la biographie}

11 Le film biographique est un genre que l'on peine à déterminer. On le dit hybride (Moine 2011). On lui attribue un modèle énonciatif, une structure narrative commune, mais des esthétiques variées (les genres surdéterminés, la comédie musicale, le film noir, etc., sont peu usités ${ }^{4}$. De plus, le biopic a eu mauvaise réputation, puisque l'industrie du cinéma en a produit beaucoup et à peu de frais, pour la télévision notamment (Custen $1992: 2$ ).

12 Écrire la vie d'une personne suppose une médiation significative (sélection, organisation, interprétation). La représentation filmique d'une vie lui octroie plus de réalité apparente que l'écrit, en vertu de l'impression de réalité qui est le propre du cinéma. Cette transposition d'une vie sur l'écran respecte habituellement les règles de vraisemblance du biopic, celles des genres secondaires convoqués et celles, plus largement, de la fiction. L'authentification qu'encourage ce type de représentation incite à y voir une fiction documentée, mais la part de fictionnalisation est substantielle. Cette activité filmique soumet la personne et le document (ou le fait) à une reconstitution de ce qui a été et à une 
fabrication de ce qui aurait pu être, par lesquelles intervient une part plus ou moins grande d'invention. Pour ce faire, un film emprunte la plupart du temps les usages d'écriture (cadrage, montage) et les modes narratifs de la fiction. À même l'image se donne à voir une perception, par laquelle se déduit une "pensée à l'œuvre » ou «en écriture ", dirait Roy (1999: 197, 199). Si cette perception dans l'image peut être « oubliée » (Roy 2015), surtout au cinéma, elle n'en est pas moins en perpétuel exercice. On peut être d'avis que l'image est un représentant et tient lieu d'une vie, mais il faut reconnaitre qu'elle tient d'abord ou simultanément lieu d'un ouvrage de signification. Cette admission importe sans doute plus dans le cas de films historiques, car l'écriture se remarque, eu égard à des événements et figures qui ont jalonné l'évolution des sociétés, et parce que le spectateur y sera attentif. Considérant ce qui précède, le spectateur serait en peine de trouver dans un biopic une transposition biographique puissamment objective. Il pourrait davantage apercevoir le travail d'écriture à l'œuvre, qui tient à divers motifs et usages qui seraient caractéristiques de ce genre, le biopic.

Les films classiques feraient une hagiographie des figures masculines reconnues pour leur excellence dans un domaine prestigieux, mais, d'après Moine (2010: 277), au moment de représenter des femmes, ils opteraient pour des «[...] rôles auxquels les femmes ont été historiquement cantonnées [... Ils] utilis[e]nt [...] leur personnage principal comme objet du spectacle [...], et [...] jou[e]nt sur l'aura sulfureuse des femmes célèbres [...]». On conçoit que le genre ait connu une évolution, du mode classique au postmoderne (Bingham 2010). Moine (2010: 280) note une augmentation du nombre de biopics féminins depuis les années 1980, mais estime que plusieurs d'entre eux «[...] emprisonnent [...] leur héroïne dans un schéma tragique [...] : les femmes exceptionnelles restent des victimes, qui paient leur talent au prix de souffrances [...] spectaculaires». Pour ne pas verser dans une vaine dénonciation, ajoutons que la tragédie demeure l'un des modes privilégiés de la fiction, célébré de longue date, et que bien souvent ce ne sont pas les victoires des personnages qui fascinent, mais leurs difficultés. On peut aussi se demander quel personnage, homme ou femme, n'est pas au service du spectacle que tout film cherche à offrir.

Le film de Parker reprend à son compte des parts biographiques associées aux deux figures péroniennes, la sainte et la mégère. Il suit la version de Main, et aussi le spectacle musical qui l'a inspiré, en présentant l'avancement du personnage par une série d'amants de service. Avant d'être sainte, Evita se montre sulfureuse et calculatrice. Le film se conclut de manière attendue par la mort tragique du personnage, auparavant soumis aux souffrances causées par son cancer. Il reprend le schéma typique : « enfance difficile [sur laquelle il insiste peu], débuts compliqués, ascension fulgurante, succès à gérer, mort (avec ou sans rédemption) » (Moine $2011: 25$ ). Il souligne aussi la flamboyante vitalité du personnage. Peter N. Chumo II (2001: 33) remarque, à propos, qu'une esthétique de la mobilité encourage cet effet. Souvent, dans un même numéro ${ }^{5}$, le personnage passe d'un lieu à l'autre grâce à ses déplacements dans l'espace et à un montage rapide, qui n'assure pas toujours de liaisons entre ces lieux hétérogènes par les moyens usuels de la fiction. Eva bouge presque constamment, et le film lui oppose, devant elle ou tout autour, les militaires, bourgeois et aristocrates, exagérément immobiles ou se déplaçant comme une masse rigide. Si le film contraint l'héroïne à un schéma classique, il n'en fait pas moins une éblouissante figure de pouvoir et d'action. Savigliano (2002: 350) remarque même que la danse métaphorise la mobilité sociale de l'héroïne, que, par ailleurs, le récit décrit de façon assez elliptique. 
15 Traçant le portrait du biopic classique français, Moine pourrait aussi bien prendre pour exemple certains aspects d'Evita. D'après l'auteure (2010: 273), le mode classique «[...] traite son héros comme un monument, dont l'éminence est donnée dès le début, et développe une mise en scène théâtrale construite autour d'un personnage-acteur dominant et souvent placé en position d'orateur ». Certaines scènes d'Evita servent même de « vignettes hagiographiques » (273) : séquences en accolade où Evita offre des cadeaux à ses descamisados, scènes où elle s'adresse au peuple du haut de la Casa Rosada, etc. Dès l' incipit, la monumentalité d'Evita est affirmée. Le début du film présente l'enfant pauvre Eva pour magnifier, par contraste, l'éminence du personnage historique. Le générique est accompagné de la bande-son d'un film (dans le film) projeté dans une salle de cinéma. Interrompant la projection, un responsable annonce sur scène la mort d'Evita. Suit la comparaison de deux funérailles : celles, modestes, du père d'Eva, agrémentées du thème musical joué en sourdine et sommairement arrangé ; celles, pharaoniques, de Santa Evita, déployées par la pleine orchestration du thème. La composition des images accentue le contraste: d'une part, un plan large, statique et dépouillé, au point de vue lointain et horizontal, perpendiculaire à la procession funéraire menée, de gauche à droite du cadre, par un char à chevaux; d'autre part, un plan large et fixe, plus court, au point de vue surélevé et en plongée, face à une rue encadrée d'immeubles majestueux où s'avancent des véhicules officiels et des militaires. Dans un cas, le mouvement linéaire des personnages et la désertion spatiale aplatissent l'image ; dans l'autre, le déplacement des personnages en profondeur (vers la caméra), les immeubles, les rangées immobiles de soldats et la foule qui longent la rue accentuent la perspective et, par accumulation, la densité de l'image. Ce second plan est chassé par un autre, où le point de vue a été déplacé de façon à massifier la foule et les soldats en mouvement, puis par un autre où une contre-plongée fait voir une effigie, devant laquelle tombent des confettis. Suivent plusieurs plans de réaction (des femmes émues). Avant même de montrer Eva Perón, le film en fait un monument et un spectacle. Le personnage, s'il est ramené à un sort tragique, n'en est pas pour autant une victime de l'histoire ou un simple poncif familier du biopic au féminin: c'est un être plus grand que nature, ennobli, même, par sa souffrance. Le film a tôt fait de convaincre le spectateur que le récit à venir sera celui d'une transformation de l'ordinaire en extraordinaire.

Evita associe un contenu à caractère historique et une forme de l'expression filmique qui sont tous deux spectaculaires. Clara Laurent le remarque, une mise en scène et une exhibition constantes entretenaient le règne d'Evita. C'est à l'occasion d'allocutions, où apparaissent nombre de plans des réactions du public, ou du Rainbow Tour, tournée « [...] mise en scène comme celle d'une pop star » (Laurent 2011: 133), que le film illustre la politique-spectacle de l'héroïne. Sa mort elle-même est mise en scène de sorte à exalter son importance. L'incipit prépare le spectateur à cette mort digne et symbolique; les scènes finales y reconduisent. À l'église, Evita ressent une douleur qui la jette au sol et un montage parallèle lie son mal à celui du peuple : la communion que reçoit Evita alterne avec le massacre d'une foule de contestataires. Le film avait jusque-là manifesté à plusieurs reprises ce lien entre la femme d'État et le peuple; cette scène le consolide et lui donne la puissance du symbole: elle n'est plus seulement femme d'État ou femme du peuple, elle est le peuple lui-même (voire l'État, lui-même). Elle mourra alors qu'une foule solennelle la veille sous sa fenêtre. Enfin, avant cette mort, des scènes intimes admettent la complicité et l'amour mutuel entre Evita et Juan Perón: un trompe-l'œil ? Aussi émouvantes soient ces scènes, une ambiguiité demeure concernant la véritable nature de 
leur relation. Main y voit une alliance stratégique. Dans son journal de tournage, Madonna (1996:174-188) rapporte ce récit troublant qu'on lui a confié :

Quand Evita était très malade et confinée à son lit, Perón interdit toute visite [...]. Lui-même la visitait rarement, car il ne pouvait supporter l'odeur de sa chambre, de son corps, de son cancer. [...] une nuit, Eva se réveilla d'un cauchemar et courut à la chambre de son mari, apeurée. [...] il la sentit et hurla: «Sors de ma chambre! Sortez cette chose d'ici ! » [...] Perón savait à quel point Evita était cruciale pour sa popularité et ce fut lui qui décida, avant qu'elle meure, d'exhiber son corps après sa mort. [...] il parla à un entrepreneur de pompes funèbres et ils décidèrent de commencer à l'injecter de mixtures chimiques qui préserveraient ses organes et sa chair ${ }^{6}$.

17 D'après Fraser et Navarro (1996: 157), ce genre de récit fait partie de nombreux mythes entourant cette période et la relation des Perón - seule une courte scène du film y fait allusion, où Eva refuse inexplicablement d'accueillir Perón dans sa chambre. Si le film choisit en définitive la version romantique, qu'il rehausse par ces scènes de veille - et Evita devient martyre -, il n'évacue pas toute trace d'ambiguïté, bien au contraire.

\section{Spectacle et distanciation critique}

18 Le récit n'insiste pas sur des intrigues politiques jouées à huis clos. Il privilégie plutôt les opérations visibles de la politique: discours, rencontres et cérémonies, interventions publiques, etc. Une telle représentation de la politique est plus cinématographique et convient au genre de la comédie musicale - ouvertement spectaculaire -, à tel point qu'aux yeux des spectateurs contemporains il passe pour factice. Intermédiale, la comédie musicale intègre la frontalité de la scène. La stabilité diégétique dont les spectateurs ont l'habitude - un monde fictionnel se donnant pour vrai - est soumise à un autre régime formel - un monde fictionnel se donnant pour ludique. Si, comme Labé (2011 : 42), l'on peut émettre l'hypothèse que le «spectateur [du biopic] est censé ne pas avoir à douter des faits qui lui sont racontés ", il faut aussitôt s'étonner que l'on puisse choisir le mode de la comédie musicale pour raconter la vie d'une personne (qui n'était pas reconnue pour sa pratique artistique). Puisque ce mode permet la distanciation, en raison de l'étrangeté mutuelle des formes artistiques conviées - chant perpétuel et réalisme dramatique -, les attentes du spectateur risquent d'être subverties, de même que l'« ancrage référentiel» (43). Le recours au régime particulier de la comédie musicale impose des normes auxquelles le spectateur du biopic n'est pas acclimaté et qui pourraient nuire à la vraisemblance (ou à tout le moins forcer la création d'un nouveau vraisemblable) : le dialogue chanté, l'organisation spatiale, qui peut loger un chœur ou des spectateurs intradiégétiques, ou encore la frontalité - Evita essayant costume après costume, se tournant devant une glace, ou Che s'adressant à la caméra. Le film fait appel à ce type de marques énonciatives, des interpellations. Un biopic habituel n'emploierait pas ces moyens ostensibles, mais intégrerait à la diégèse les potentiels points de rupture par le recours à la psychologie des personnages, à une causalité narrative, ou à une quelconque justification intradiégétique: par le recours, donc, à une "énonciation diégétisée ", comme l'indiquait Casetti (1990 : 55). Dans Evita, la suture de la fiction n'est pas toujours assurée ou maintenue. Les personnages y chantent plutôt qu'ils ne parlent ; les limites et usages du biopic sont soumis aux pressions de la comédie musicale. À l'heure où ce genre est rare, on ne manquera pas de remarquer l'audace d'un tel choix artistique. $\mathrm{Au}$ surplus, il réfère obliquement à l'organisation politique du régime Perón (et des 
régimes totalitaires en général), ou en fait figure. Il s'agit, pour Laurent (2011: 133), du "[...] parti pris interprétatif des auteurs du film: le "règne" de la prima donna du péronisme fut un show, un spectacle permanent, scénarisé, mis en scène [...]». L'évoquant, le personnage de Che scande : "Quel cirque! Quel spectacle!», ou encore : «La meilleure performance en ville était celle de la foule. »

19 À cette utilisation du spectaculaire pour thématique, figure ou esthétique, le film ajoute, comme autre interférence, un discours critique sous-jacent, pris en charge, en certains cas, par le montage et le récit. Une séquence alterne deux séries de plans: la montée politique de Perón, grâce à des pratiques déloyales, et l'ascension d'Eva dans le milieu des arts (Chumo II 2001 : 34). Le discours critique est endossé par Che. Partie prenante du triumvirat à la tête du film, cet être mystérieux apparaît avant les deux autres, dans la salle de cinéma où la mort de l'hérö̈ne est annoncée. Après les processions funéraires, on le retrouve dans un bistrot, puis marchant près de la foule amassée pour voir passer le cercueil. Il chante en s'adressant à la caméra : « Mais qui est cette sainte Evita? Pourquoi cette hystérie, ces hurlements de souffrance ? [...] Aussitôt que la fumée des funérailles se sera dissipée, nous verrons bien [...] qu'elle n'a rien fait pendant des années. » Ses irruptions se multiplient au cours du film, de même que les identités narratives qu'il revêt provisoirement : il est tantôt spectateur anonyme, serveur, assistant d'Eva, tantôt mort, puis vivant, etc. Il s'adresse au spectateur et conduit certaines parties du récit, agissant alors comme narrateur. Ses sarcasmes mettent en doute la bonne foi de la politicienne. Quant à elle, la scène où tous les deux valsent ensemble précise le discours critique sous-jacent. Il s'agit d'une scène imaginaire, à laquelle les deux personnages participent après avoir perdu connaissance : Che a été abattu par la milice pendant une manifestation; Evita est hospitalisée. On l'anesthésie et la scène commence: les protagonistes se font face, puis se mettent à danser résolument en échangeant questions accusatrices (de Che) et ripostes déterminées ( $\left.\mathrm{d}^{\prime} \mathrm{Eva}\right)$. La scène donne lieu à une exposition des reproches antipéronistes: "Comment peux-tu prétendre être notre sauveuse quand tes opposants sont piétinés, torturés ou tout simplement disparaissent ? [...] Comment peux-tu avoir la vue si courte, ne jamais voir plus loin qu'une semaine ou deux à la fois, ne contempler aucun rêve impossible?" lance Che, référant aux interventions dites de courte durée et à faible portée sociale qu'Evita a multipliées. Celleci rétorque qu'elle ne peut à elle seule transformer une tradition politique systémique, dont rendent compte ces exactions, et qu'une action sociale à petite échelle vaut mieux que d'essayer de résoudre des problèmes complexes, insurmontables.

Laurent (2011 : 135) conclut que cette critique empiète sur la relation entre le spectateur et le film comme objet de divertissement : «[...] Evita adopte [...] un point de vue fort, par le biais de ce coryphée impitoyable dans son analyse des événements. La distanciation est donc quasi constante, le spectateur ne pouvant jamais tout à fait se laisser aller au plaisir d'assister à un spectacle grandiose. » Les justifications offertes par Evita aux accusations de Che sont peu convaincantes, sinon la dernière, irréfragable : elle s'affaisse au sol et demande à son alter ego, son opposant : «à quoi peut servir le cœur le plus vaillant dans un corps qui se dérègle ? » À la fin, où le film reprend les scènes funéraires qu'il avait présentées au début, se trouve un autre rappel, chanté par un chœur alors que Che et Perón se font face à leur tour, auprès du cadavre d'Evita : « yeux, coiffure, visage, image ; tout doit être préservé; vie figée, exposée à jamais; rien de moins que ce qu'elle méritait. » Faute de connaître les faits entourant l'embaumement du corps, le spectateur entendra ces paroles mystérieuses comme prolongeant une figure tramée au long du 
film : spectacle, mise en scène (jusque dans la mort). Le spectateur qui sait l'outrage commis par la manipulation du cadavre entendra dans le dernier vers une triste ironie, un point d'orgue à la pensée critique du film.

21 Par divers moyens, cette pensée critique entraverait donc non seulement un certain plaisir spectatoriel associé au biopic de divertissement (l'attestent les critiques de l'époque et le tiède accueil populaire réservé au film), mais aussi la fiction elle-même ; cette pensée critique établit les conditions d'un autre plaisir spectatoriel et d'un autre régime fictionnel, entremêlant imaginaire et réalité. Le biopic habituel, d'après Custen (1992: 3), «intègre [...] des épisodes historiques disparates [...] en une "vision hollywoodienne de l'histoire" quasi monochrome ${ }^{7} »$. Or, en dépit des efforts consentis à l'uniformisation de la représentation - le film privilégie en effet la figure de la sainte amoureuse à celle du potentat opportuniste -, la pensée critique aménagée dans le film, qui relève davantage $d u$ commentaire que du récit, impose au spectateur un questionnement à caractère éthique. Comme nous l'avons dit, l'histoire d'Eva Perón est truffée d'incertitudes, elle se présente en versions (Dujovne Ortiz 1995: 25-26). Cette multiplicité, que l'autobiographie officielle d'Evita a stimulée, puisqu'elle contient des réécritures significatives de son propre passé (Fraser et Navarro 1996: 5), arrange en amont la biographie, la vie d'Eva Perón sur le mode mythique. Par l'ambivalence politique et identitaire qu'il cultive, par le genre de la comédie musicale, inusité pour le mode sérieux du biopic à caractère historique, le film de Parker contribue à la condition mythique d'Evita - tout en s'en servant - plus qu'il n'invite à des rectifications. Puisque les questions que pose le film restent sans réponse, la pensée critique n'y est pas démystificatrice, mais participe, elle aussi, du mystère en l'exposant.

\section{Identité en différé}

La chanteuse et actrice Madonna dispose d'un statut singulier qui connote son interprétation d'Eva Perón. À travers ses nombreuses incarnations, ses pratiques artistiques et médiatiques, Madonna pourrait revendiquer, d'après Sécardin, une position auctoriale postmoderne, dans la mesure où elle procède par collage, citations et remplois; chaque nouvelle référence s'ajoute à une identité fluctuante et libre (ou plus libre) - son interprétation d'Eva Perón entraîne une amplification du caractère mythique des deux femmes, postulera-t-on ici. Savigliano (2002 : 346) critique la proposition du film à ce chapitre. Pour elle, le casting de Madonna occasionne un affaiblissement de la dimension politique du film, en même temps qu'un accroissement de sa valeur marchande. L'auteure souligne à ce sujet la célébrité de Madonna comme produit de consommation courante (ce qui serait, d'après elle, un tort) et son identité qu'elle dit souple mais superficielle, qui produiraient une image d'Evita vidée de toute spécificité. C'est privilégier une perspective politique. Or, si le film raconte l'histoire d'une personnalité politique, son intérêt est ailleurs. La lecture politique non seulement n'est pas la seule possible, mais il est probable qu'elle n'aura pas été celle de la plupart des spectateurs ni celle des créateurs.

Plusieurs penseurs se sont penchés sur le phénomène des stars en examinant l'imaginaire culturel qu'elles nourrissent (que l'on songe à l'ouvrage bien connu d'Edgar Morin sur les stars ou, du côté anglophone, aux travaux de Parker Tyler ou de Camille Paglia). Dans une perspective similaire, certains considèrent la star qu'est Madonna comme une figure mythique à l'esthétique postmoderne. Se donnant en un spectacle continu, elle en vient à 
incarner l'idée même du spectaculaire, puisant à la culture populaire et multipliant les images, dont plusieurs sont d'ores et déjà mythiques (et remployées par d'autres). Entre réalité et fantasme, entre vérité et mensonge, la référence du signe «Madonna » est en perpétuel différé. Madonna est alternativement ou concurremment Monroe / Dietrich, vierge / putain, féminin / masculin, dominée / dominante, etc. Sécardin (2002: 68) remarque que son régime est celui de la feintise. La pratique ou l'identité auctoriale de Madonna est ainsi tendue entre les indices de subjectivité, affirmés puis biffés ou remplacés, et les diverses références que ces indices connaissent - en préface de son livre Sex, une exploration de son imaginaire sexuel, elle écrit : «Rien dans ce livre n'est vrai. J'ai tout inventé ${ }^{8} »$ (Madonna $1992: 2$ ) ; les images et les textes contenus dans cet ouvrage ont pour références la culture pornographique et sadomasochiste, ou encore la forme littéraire du journal. Si aucune œuvre n'échappe à ses propres références, dans celle de Madonna ces attaches esthétiques et culturelles sont en général admises, et l'artiste en joue le jeu. Si bien, écrit Sécardin (2002:68), que « [...] le personnage est aléatoire et n'est pas soumis à un régime continu [...] ». L'énonciation est potentiellement feinte ou vraiesemblable. Elle promeut la distanciation, l'ironie, l'esthétique de la duplicité ou du jeu, l'emploi de personnages substituables (comme les costumes de scène), véritables personas, et l'intertextualité. L'identité virtuelle, conclut Sécardin (2002: 78), en vient à valoir pour l'existence réelle. Chaque image qu'offre la chanteuse en appelle d'autres, passées ou à venir.

24 C'est ainsi que Sécardin (2002: 66) situe l'œuvre de Madonna dans le champ de l'autobiographie postmoderne, y constatant la neutralisation de la référence à une réalité, à une identité aisément discernables : «Le "je" postmoderne[,] qui n'a de réalité que l'effet de sa représentation, se trouve [en quelque sorte ou provisoirement] séparé du "je" qui l'énonce. » L'incarnation de Madonna en Evita vaut par elle-même, et a d'ailleurs servi à la promotion du film. Les journalistes mentionnaient pour la plupart les ressemblances entre les deux femmes: la légende entoure l'arrivée de chacune dans la grande ville; chacune a survécu à la mort d'un parent pendant l'enfance, etc. Madonna (1996) ellemême soutient cette idée de l'incarnation. Évoquant la lettre qu'elle a écrite au réalisateur pour le convaincre qu'elle ferait la meilleure candidate pour le rôle, elle précise : «Je peux honnêtement dire que je n'ai pas écrit cette lettre seule. C'était comme si une autre force menait ma main le long de la feuille ${ }^{9}$. Puisque Madonna s'approprie l'identité du personnage, le film s'avère doublement biographique, pourrait-on avancer.

\section{Le biopic postmoderne}

Ce différé entre le sujet, l'auteur et l'objet caractériserait le mode postmoderne du biopic. Evita et W.E., un film réalisé par Madonna et paru en 2011, en sont des exemples. C'est aussi le cas, de façon encore plus marquante, de I'm Not There (2007), où plusieurs acteurs de sexes et d'âges différents se partagent le rôle de Bob Dylan. Le contenu référentiel s'y montre si incertain que la substance biographique du film s'évanouit. Ces films font penser que, «[a]u cœur du genre biopic, se logent les subtilités et les paradoxes du réalisme dans la fiction cinématographique » (Labé 2011: 42). On tient la référence (à la réalité, à l'histoire) pour essentielle dans le biopic, mais elle compte dans n'importe quel film de fiction. Cependant, le réalisme, comme tout autre effet filmique, a pour première condition une écriture et non un transfert de réalité. Autrement dit, le film procède 
d'abord d'une fabrication, ce sur quoi un certain type de biopics est susceptible d'attirer l'attention.

Comme nous l'avons vu, Evita s'écarte des formes représentationnelles généralement associées au film documenté. Puisqu'Eva Perón a le statut d'une figure mythique, eu égard à la refiguration historique dont elle a fait l'objet depuis sa mort et même avant, la réalité factuelle est contrainte à une réduction, et ce, au profit d'un imaginaire qui, inversement, l'agrandit. Le film ne respecte que sommairement les conventions du biopic en raison de la distanciation qu'il exerce. En un sens, Madonna est aussi mythique, et son identité, en perpétuelle reconfiguration, se superpose à celle d'Evita, quitte à l'absorber. Plutôt qu'à un ordre de factualité, la vérité de l'une et de l'autre se rapportent ainsi à un ordre de croyance, celui du film.

Le même régime de signification se profile dans le film W.E., pour lequel Madonna (la réalisatrice) emprunte la voie du biopic. Elle a maintes fois exprimé sa fascination pour le personnage d'Evita; dans W.E., une autre femme politique controversée, Wallis Simpson, fascine une riche Américaine. Le film intègre à son esthétique des procédés qui autorisent une lecture critique. Le récit est celui d'une enquête que mène l'héroïne sur la duchesse de Windsor. Sa structure narrative, alternant entre présent et passé, invite le spectateur à déduire des liens entre les personnages et les événements d'époques différentes. Par moments, la représentation se fait à vue : des séquences documentées ou biographiques paraissent résulter de l'imagination renseignée de l'héroïne. Si, dans Evita, la présence de Madonna à titre d'interprète redoublait le personnage historique, dans W.E., le récit confectionne lui-même la doublure puisqu'il met en relation deux temporalités, deux espaces géographiques, deux personnages: l'un historique, l'autre fictif. Pour Kavka (2014), W. E. opère plus sous le mode de l'autobiographie (un mémoire-écran) que de la biographie puisque le procédé narratif permet d'illustrer l'étrange dualité de l'auteure, qui est à la fois la femme fascinante et la femme fascinée.

\section{La pensée du biopic}

La réalité historique ne concerne pas que des faits, mais aussi des perceptions et une relation aux faits, aux mémoires, une memoria : «Participant de l'imaginaire, où s'exerce l'imagination, le réel est le sujet d'écritures variées. Celles qui le concernent se constituent en véritable memoria, réalisée à partir d'un exercice phénoménologique de discernement, d'interprétation, etc. » (Roy 2017). A contrario, les relativistes se trompent gravement lorsqu'ils évacuent les faits ou l'objectivité. Les biopics, c'est-à-dire, comme nous l'avons suggéré, des films documentés plutôt que documentaires, enchevêtrent les nombreux fils de l'histoire d'une vie, dont ils documentent les faits aussi bien que l'imaginaire que ces derniers ont alimenté. Les biopics ont un statut particulier, eu égard au degré de créance qu'ils invitent le spectateur à leur accorder (c'est la vie de...) et à la fictionnalisation qu'ils lui font accepter. Ils sont le fruit d'un travail de l'imagination, duquel toutefois le fonds documentaire n'a pas été neutralisé. Ce sont, donc, de vrais mythes.

$\mathrm{Au}$ mythe Evita correspond une identité historique. Par le film de Parker, son association à Madonna ajoute une image de plus aux visages que revêt Evita dans l'imaginaire des sociétés. (Cela explique sans doute les réactions mitigées, voire hostiles, de certains Argentins au casting de Madonna : ils ne souhaitaient pas voir l'image de cette femme se 
superposer à celle de leur idole.) Inversement, Madonna a profité, sur le plan du sens, d'une image supplémentaire, déjà traversée d'une mythologie socioculturelle importante. L'identité de l'une et celle de l'autre fondent en ce film le site d'un imaginaire culturel partagé.

À tout prendre, le film montre moins la réalité biographique événementielle ou factuelle qu'il ne témoigne de l'attachement à cette figure historique qui motive une fictionnalisation de la biographie. Ou, pour emprunter cette fois la proposition de Ricœur ([1985] 1991: 252), le film exerce davantage une "fonction de signifiance " qu'une "fonction de représentance». La spectacularité du film accroît l'effet de la fictionnalisation préalable de la personne historique. On ne saura pas plus qui a vraiment été Eva Perón, mais le film vaut pour une hypothèse. La biographie est emportée par le sefaire du film, qui, par un travail temporel (sélection, condensation, ordonnancement) et esthétique (spectacularisation, interpellation, commentaire), confirme le statut mythique du personnage, voire propose, comme solution à l'impossibilité de rendre raison de sa vie, d'en admirer l'imaginaire qu'il fait fructifier. Quant à Madonna, si l'on ne saura pas plus qui elle est vraiment, on admettra l'apport identitaire que son incarnation d'une femme mythifiée lui assure sur le plan imaginaire et artistique. Si le passé historique et biographique compte évidemment, la signifiance prime.

31 Nonobstant sa singularité, le cas Evita incite à penser que les biopics en général ressortissent aux mêmes procédés fictionnels que lui, procédés qu'en même temps l'historicisation authentifie. Il invite aussi à penser que la référence à la réalité est approximative, ou du moins que les récits factuels et imaginaires, de concert, contribuent à dresser une image de la vie d'une personne et d'une société à un point de son évolution. Cela étant, le biopic nous informe plus sur l'imaginaire social que sur des faits relatifs à l'histoire. À terme, par une réflexion sur le biopic, l'on peut admettre son statut de genre, remarquant aussi qu'il satisfait à des normes esthétiques et narratives indépendantes de la vérité historique à laquelle les films se réfèrent ou prétendent se référer. On ne saurait évaluer ces films en fonction du seul critère de fidélité à «l'histoire vraie " présumément reportée à l'écran. Ces films sont, comme d'autres, le résultat d'une confection fictionnalisante qui, d'une vie, offre un spectacle. Par là, c'est bien souvent le caractère spectaculaire de ces films, leur caractère fictionnel, qui retient l'attention - et l'intérêt -, plutôt que la vérité historique. Ils sont immanquablement fidèles à une pensée biographique - significative (elle fait naitre le sens), exemplaire (le récit d'une vie parmi d'autres), « vraie » (cette pensée est bel et bien à l'œuvre).

\section{BIBLIOGRAPHIE}

Bingham, Dennis, 2010, Whose Lives Are They Anyway? The Biopic as Contemporary Film Genre, New Brunswick, Rutgers University Press, p. 3-28.

Casetti, Francesco, 1990, D'un regard l'autre. Le film et son spectateur, Lyon, Presses Universitaires de Lyon. 
Chumo II, Peter N., 2001, “"The Greatest Social Climber Since Cinderella”: Evita and the American Success Story ", Literature/Film Quarterly, vol. 29, n 1, p. 32-36.

Custen, George F., 1992, Bio/Pics. How Hollywood Constructed Public History, New Brunswick, Rutgers University Press.

Dujovne Ortiz, Alicia, 1995, Eva Perón, New York, St. Martin's Press.

Eliade, Mircea, 1963, Aspects du mythe, Paris, Gallimard.

Flores, María, 1953, La Femme au fouet, trad. Lola Tranec, Paris, Club français du livre.

Fraser, Nicholas et Navarro, Marysa, 1996, Evita. The Real Life of Eva Perón, New York, W. W. Norton \& Company.

Kavka, Misha, 2014, « Hating Madonna and loving Tom Ford: Gender, Affect and the "ExtraCurricular" Celebrity ", Celebrity Studies, vol. 5, n ${ }^{\text {os }} 1-2$, p. 59-74.

Labé, Benjamin, 2011, « Vraisemblance et vérité : la question du réalisme et ses paradoxes », dans R. Fontanel (dir.), Biopic : de la réalité à la fiction, Condé-sur-Noireau, Charles Corlet, p. 42-47.

Laurent, Clara, 2011, « Les femmes de pouvoir du xx siècle. Evita, d'Alan Parker : Santa Evita, Santa Madonna », dans R. Fontanel (dir.), Biopic : de la réalité à la fiction, Condé-sur-Noireau, Charles Corlet, p. 130-136.

Madonna, 1992, Sex, New York, Warner Books.

Madonna, 1996, « Madonna’s Private Diaries », Vanity Fair, nº 435, p. 174-188.

Main, Mary, [1952] 1980, Evita. The Woman With the Whip, Londres, Corgi.

Martínez, Tomás Eloy, [1995] 1997, Santa Evita, Paris, Robert Laffont.

Moine, Raphaëlle, 2010, « Le biopic à la française : de l'ombre à la lumière », Studies in French Cinema, vol. 10, n 3, p. 269-287.

Moine, Raphaëlle, 2011, «Le genre biopic », dans R. Fontanel (dir.), Biopic : de la réalité à la fiction, Condé-sur-Noireau, Charles Corlet, p. 22-27.

Ricœur, Paul, [1985] 1991, Temps et récit. Tome III. Le temps raconté, Paris, Seuil.

Ricœur, Paul, [1990] 1996, Soi-même comme un autre, Paris, Seuil.

Roy, Lucie, 1999, Petite phénoménologie de l'écriture filmique, Québec, Paris, Klincksieck, Nota bene.

Roy, Lucie, 2015, Le Pouvoir de l'oubliée. La perception au cinéma, Paris, L'Harmattan.

Roy, Lucie, 2017, « Une ontologie ou une esthétique numérique ? », Sens public, 15 décembre 2017, [En ligne], http://sens-public.org/article1281.html, consulté le 24 juin 2018.

Savigliano, Marta E., 2002, « Evita: The Globalization of a National Myth », dans J. Abbassi et S. L. Lutjens (dir.), Rereading Women in Latin America and the Caribbean. The Political Economy of Gender, Lanham, Rowman \& Littlefield, p. 344-360.

Schwartz, Margaret, 2010, «Proper Corruption Index and Metaphor in Photographs of the Embalmed Corpse of Eva Perón », Framework. The Journal of Cinema and Media, vol. 51, n 1, p. 7-32.

Sécardin, Olivier, 2002, « L'autobiographie post-moderne, post-mortem: Madonna auto(bio)graphe ", L'Esprit Créateur, vol. 42, $\mathrm{n}^{\circ} 4$, p. 66-75.

Spirou, Penelope, 2011, The Musical Biopic: Representing the Lives of Music Artists in 21st Century Cinema, thèse de doctorat, Sydney, Macquarie University. 


\section{NOTES}

1. Main publie d'abord sous le pseudonyme de María Flores. Une seule édition française nous est connue, La Femme au fouet (1953). Référence sera faite aux deux éditions.

2. «[...] a compendium of the most virulent anti-Peronist gossip ». Notre traduction (idem pour toutes les citations issues de références anglaises à venir).

3. Il fut embaumé dès sa mort, puis volé par les antipéronistes, caché en Italie, rapatrié en Argentine pour servir les ambitions de la troisième femme de Perón. Ce corps et sa manipulation témoignent de la valeur symbolique de Santa Evita, vivante même après la mort (voir Schwartz 2010 : 7-32 ; Fraser et Navarro 1996). Le film de Parker se termine, pour sa part, aux funérailles et n'évoque donc pas cette partie subséquente de l'histoire du personnage.

4. D’après Moine (2011 : 26), « le mélange du biopic et de la comédie musicale, ou plus largement du biopic et du film musical » serait «l'une des [hybridations du biopic] les plus prolifiques ». Si l'on excepte le film musical, qui n'est pas une comédie musicale (il n'inclut pas de scènes chantées et dansées qui suspendent le récit, ou qui modifient le mode narratif), il faudrait noter que les comédies musicales, biographiques ou non, ont disparu de la production régulière depuis des décennies. Les comédies musicales encore produites paraissent à titre de singularités (voir Spirou 2011 : 44-46).

5. Comme dans plusieurs comédies musicales, le numéro (la performance) équivaut à la scène ou à la séquence.

6. "When Evita was very ill and confined to her bed, Peron forbade any visitors [...]. He himself rarely visited, because he could not stand the smell of her room, her body, her cancer. [...] one night Eva woke up from a bad dream and got out of bed to go to [...] Peron's room because she was scared. [...] he smelled her and shouted, "Get out of my room, get that thing out of here!" [...] Peron knew how instrumental Evita was to his popularity and it was he who decided, before she died, to have her body put on display after her death. [...] he talked to a mortician and they decided they would have to start injecting her with chemical concoctions which would preserve her organs and flesh. »

7. «[...] integrates disparate historical episodes [...] into a nearly monochromatic "Hollywood view of history." "

8. « Nothing in this book is true. I made it all up ».

9. «I can honestly say that I did not write this letter of my own free will. It was as if some other force drove my hand across the page ».

\section{RÉSUMÉS}

Plusieurs films qui se prêtent à la "biographie» laissent songeur quant à la vérité de la représentation. Le cas du film Evita intéresse ici, car il met à profit plusieurs ambiguïtés : la vie d'Evita, fortement mythifiée, comme celle de son interprète, la chanteuse Madonna. Par le mode spectaculaire de la comédie musicale, le film admet son approche ludique. L'examiner sous cet angle fait penser que tout récit biographique invente, monte, découpe le fil d'une vie, de sorte que la vérité y paraît d'abord tenir à l'interprète qu'est l'auteur ou même, dans le cas d'Evita, à la sorte d'interprète-auteure qu'est l'actrice. 
Films that lend themselves to "biography" let us ponder the truthfulness of their representation. The case of the film Evita is of particular interest, because of the several ambiguities upon which it is built: the life of Evita, largely mythologized, as is her interpreter's, the singer Madonna. The somewhat frivolous approach of the film seems acknowledged by its chosen stylistic mode, that of the musical. This perspective suggests that all biographical narratives invent, edit, cut the linear logic of a life, so that the truth first appears to be dependant upon the author-interpreter or, in Evita, on the author-performer.

INDEX

Keywords : biopic, cinema, Evita, Madonna, identity, fictionalization

Mots-clés : biopic, cinéma, Evita, Madonna, identité, fictionnalisation

\section{AUTEUR}

\section{GABRIEL LAVERDIÈRE}

Université Laval 